\title{
Antibiogram sensitivity in urinary tract infections (UTI) at el batnan medical center- tobruk - libya
}

\begin{abstract}
The aim of this study is to identify the most common bacteria that cause UTI and to rationalize antibiotic guidelines for UTI to optimize outcome and to minimize resistance at a reasonable cost to the patient and the community. In a clinical trial to identify the sensitivity/resistance pattern of ten different antimicrobial agents commonly used in the treatment of urinary tract infections (UTI) in El Batnan Medical Center (BMC), TobrukLibya, during 2003 and 2004. A total of 773 midstream urine samples from outpatients and inpatients attended Central Lab in El Batnan Medical Center (BMC) clinically suspected as having UTI, were examined for microbiological confirmation and pattern of antibiotic susceptibility by disc diffusion method. By using direct smear microscopy and routine culture methods, 8 different bacterial species were isolated from only $262(33.9 \%)$. The remaining $511(66.1 \%)$ of cases showed no bacterial growth.
\end{abstract}

Bacteriological examination of the urine samples showed Escherichia coli strains isolated in $106(40.4 \%)$ of cases with UTI, Klebsiella in $59(22.5 \%)$, Staph epidermidis in $35(13.3 \%)$, Staph aureus in $32(12.2 \%)$, Proteus spp in $12(4.5 \%)$, while $8(3 \%), 6(2.3 \%), 4(1.5 \%)$ of cases showed Pseudomonas, Entrococci and Corynebacteria respectively. The study of the antimicrobial sensitivity/resistance pattern of ten different antimicrobial agents commonly selected in the treatment of UTIs, indicated that the antimicrobial drug of choice for treating UTI caused by Escherichia coli strains, Klebsiella, Staph. epidermidis, Staph. aureus, and Proteus spp. should include Ciprofloxacin (80.2\% sensitivity), or Amoxicillin (70.2\% sensitivity), or Nitrofurantion (64.5\% sensitivity), or Ceftriaxone $(61.8 \%$ sensitivity). Meanwhile, for treating UTI produced by Entrococci, the antimicrobial drug of choice is Amoxicillin (70.2\% sensitivity), Naldixic acid (60.3\% sensitivity), and Ampicillin (9.2\% sensitivity), while for treating Pseudomonas spp, the drug of choice should be member of Fluoroquines group as Ciprofloxacin ( $80.2 \%$ sensitivity), and Sulphamethoxazol + Trimethoprim (Co-trimoxazole) (26.3\% sensitivity).

Keywords: urinary tract infections (uti), significant bacteruria, disc diffusion method, antibiotic sensitivity/resistance, empirical treatment
Volume 4 Issue 3 - 2017

\author{
Mahmoud D Dabobash,' Makram Fahmy \\ Attla,' Muftah Elgarba,' Azza SA Menshawy' \\ 'Laboratory medicine and Pathology, El Batnan Medical Centre \\ (BMC), Libya \\ ${ }^{2}$ Faculty of Medicine, Ain Shams University, Egypt
}

Correspondence: Muftah Elgarba, Faculty of Medicine, Omer Al Mukhtar University, Al-Bida, Libya,

Email elgarba_uro@yahoo.com

Received: July 24, 2016 | Published: March 28, 2017

\section{Introduction}

Antibiotics are used to prevent infection and to treat patients with proven or suspected infection. The aim is to administer a safe and cost effective dose of antibiotic that will eliminate the infecting or potentially infecting organism. Antibiotics are widely used, contributing to $35 \%$ of all prescriptions in health care facilities. Overuse of antibiotics results in bacterial resistance not only to the antibiotic prescribed, but often to other antibiotics in the same classes or groups. The abuse or misuse of antibiotics is costly because it leads to the emergence of antibiotic resistance among microorganisms in the health care facility environment as well as in the patients. When infections from Antimicrobial Resistant Organisms occur, there is increased mortality, especially among those with underlying diseases or multi organ failure.

$\mathrm{In}^{1}$ the past $30-50$ years, the natural history of urinary tract infection (UTI) has changed as a result of the introduction of antibiotics and improvements in healthcare. This change has contributed to uncertainty about the most appropriate and effective way to manage UTI and whether or not investigations and follow-up are justified. UTI is a common bacterial infection in children, found in up to $5 \%$ of all febrile children under the age of 2 years presenting to emergency rooms (Van der Voort 1997) and with an incidence of $0.43 / 1000$ patients per year in general practice Nordenstam GR, et al. ${ }^{2}$ A population-based study from the UK based on referral data collected over 4 years suggested that $11.3 \%$ of females and $3.6 \%$ of males will have had a UTI by the age of 16 Coulthard et al. ${ }^{3}$ Studies suggest that UTI has the incidence of first time and recurrent episodes of UTI in general practice ranges from $0.6 \%$ and $1.1 \%$ in boys and girls, respectively, aged under 1 year while it changes to $0.2 \%$ and $1.4 \%$ for boys and girls, respectively, aged between 5 and 14 years Jadresic $\mathrm{L}$ et al. ${ }^{4}$

Coulthard et al..$^{5}$ showed that an education model when combined with prompt diagnosis and access to a nurse led UTI service increased the pickup rate of patients appropriately diagnosed with UTI by four times that of the control group. Different antimicrobial regimens have been used in the treatment of UTI, differing in both type and duration of treatment. However, there is no consensus as to which antimicrobial should be used and how long treatment should be continued. In this study, we try to achieve more consistent clinical practice, based on accurate laboratory diagnosis and effective management hence reducing the emergence of other resistant forms of bacteria and minimizing the costs due to unnecessary antibiotic abuse. 


\section{Materials and methods}

A total of 772-midstream urine samples sent for microbiological study at the Central Lab. of El Batnan Medical Center, were studied during four months (October and November 2003 and 2004). Patients were clinically diagnosed according to criteria already established for UTI diagnosis Klein RS. ${ }^{6}$

\section{Collection of specimens}

A clean midstream urine sample is the recommended method for urine collection. In babies and infants urine samples were collected in sterile self adhesive plastic bags Liaw LCT et al. ${ }^{7}$ When it was not possible or practical to collect urine by non-invasive methods, catheter samples $23(2.97 \%)$ or suprapubic aspiration (SPA) $7(0.9 \%)$ used. Before SPA is attempted, ultrasound guidance should be used to demonstrate the presence of urine in the bladder. Samples were sent to the laboratory within one hour after voiding when possible or kept refrigerated at $40 \mathrm{C}$ to avoid multiplication of bacteria in urine resulting in false significant bacteruria Abla M El-Mishad. ${ }^{8,9}$

\section{Used laboratory technique}

Direct smear microscopy and routine bacterial cultures included the following materials

i. Clean containers $\rightarrow$ to collect stool specimen

ii. Bacteriological loops $\rightarrow$ for inoculation of culture media.

iii. Swabs $\rightarrow$ used for spreading of colonies on nutrient agar for "antibiotic sensitivity test"

iv. Forceps $\rightarrow$ for putting antibiotic discs on nutrient agar for "antibiotic sensitivity test".

Direct smears from urine deposits after centrifugation were stained with Gram's stain.

\section{Culture media}

MacConkey agar (European pharmacopoeia). For study of coliform organisms.

Laboratorios CONDA La Forja, g- 28850 Torrejon de Ardoz Madrid (Spain)

Preparation: Suspend 50 grams of the medium in one litre of distilled water. Mix well until a uniform suspension is obtained. Heat with frequent agitation and boil for one minute until completely dissolved. Cool to $45^{\circ} \mathrm{C}$ and pour in Petri dishes. Allow the plates to solidify and place them upside down to avoid excessive moisture on the surface of the medium.

\section{i. Mueller-Hinton agar (OXOID CM0337) \\ ii. Typical formula $(\mathrm{g} / \mathrm{l}) \mathrm{pH} 7.3$ at $25^{\circ} \mathrm{C}$ \\ iii. Beef, dehydrated infusion from 300.0}

iv. Casein hydrolysate 17.5; Starch 1.5; Agar 17.0

\section{Oxoid Ltd., basingstke, hampshire, england}

Preparation: Suspend 38 grams of the medium in one litre of distilled water. Bring to the boil to dissolve the medium completely. Sterilize by autoclaving at $121^{\circ} \mathrm{C}$ for 15 minutes.

I. COLUMBIA agar base (OXOID CM0331)
II. Typical formula (g/l) $\mathrm{pH} 7.3$ at $25^{\circ} \mathrm{C}$

III. Special peptone 23.0; Starch 1.0; Sodium chloride 5.0; Agar 10.0

\section{Oxoid Ltd., basingstke, hampshire, england}

Preparation: Suspend 39 grams of the medium in one litre of distilled water. Bring to the boil to dissolve the medium completely. Sterilize by autoclaving at $121^{\circ} \mathrm{C}$ for 15 minutes. Cool to $50^{\circ} \mathrm{C}$ and add $5 \%$ sterile defibrinated blood. For identification and quantitation, all bacteria were cultured on MacConkey's agar medium. Blood agar medium, Nutrient agar media and incubated over night at $37^{\circ} \mathrm{C}$. The colonies are further identified by morphology, Gram staining and API biochemical reactions. The quantitation of bacteria in urine samples was done using calibrated loops for cultivation Jawetz, et al. ${ }^{10}$

Antibiotic discs $\rightarrow$ for antibiotic sensitivity test

Were supplied by Himedia Laboratories PVT. LTD. 23, Vadhani Ind, Est., LBS Marg, Mumbai- 400086, India, and Oxoid LTD., DasinGstoke, Hampsttire. England.

Antibiotics used (with their abbreviations):

1) Ampicillin “AMP" 2) Amoxicillin "AMC"

3) Nalidixic acid "NA" 4) Ceftriaxone "CRO"

5) Suplha methoxazol + trimethoprim "SXT" 6) Cephalothin "KF"

7) Ciprofloxacin "CIP" 8) Nitrofurantion "F"

9) Chloramphenicol "C" 10) Doxacycline "DO"

\section{Susceptibility tests}

Disc diffusion method of "antibiotic sensitivity test"

Steps: Emulsify several colonies of the test organism in nutrient agar and incubate over night at $37^{\circ} \mathrm{C}$.

Put antibiotic disc on plate by the forceps and incubate the plate aerobically at $37^{\circ} \mathrm{C}$ over night.

In the next day, take the readings for antibiotic sensitivity and resistance.

The reaction of the test organism to each antibiotic is reported as follow:

i. Sensitive: Big zone of inhibition.

ii. Intermediate: intermediate zone of inhibition.

iii. Resistant small or no zone of inhibition.

\section{Results}

The study was done on a total of 773 urine samples from patients clinically diagnosed as UTI in Al-Batnan Medical Center, during four months October and November 2003, October and November 2004. Out of the 773 cases examined microbiologically 262 showed positive growth on bacterial cultures, $173(66 \%)$ of them were children under 12 years old. UTI patients with no bacterial growth, represent 511(66.1\%), meanwhile cases with positive bacterial growth, represent 262(33.99\%) (Table I). The isolated pathogenic bacteria causing UTIs were Escherichia coli strains isolated in $106(40.46 \%)$ 
in cases with UTI, Klebsiella in 59 (22.52\%), Staph epidermidis in $35(13.3 \%)$, Staph aureus in 32 (12.12\%), Proteus spp in $12(4.5 \%)$,

while $8(3.05 \%), 6(2.29 \%), 4(1.53 \%)$ of cases were produced by Pseudomonas, Entrococci and Corynebacteria respectively Table 2.

Table I Frequency (\%) of cases with positive cultures

\begin{tabular}{|l|l|l|l|}
\hline Month & No of Cases with UTI & $\begin{array}{l}\text { No of Cases with Positive } \\
\text { Culture }\end{array}$ & Percentage (\%) \\
\hline October 2003 & 197 & 58 & $29 \%$ \\
November 2003 & 150 & 62 & $41 \%$ \\
October 2004 & 244 & 91 & $37 \%$ \\
November 2004 & 182 & 51 & $28 \%$ \\
\hline Total & 733 & 262 & $100 \%$ \\
\hline
\end{tabular}

Table $\mathbf{2}$ This table shows the frequency $(\%)$ of pathogenic bacteria in positive culture cases

\begin{tabular}{|l|l|l|}
\hline Pathogenic Bacteria & No of Positive Culture Cases & Frequency (\%) \\
\hline Escherichia coli strains & 106 & $40.46 \%$ \\
\hline Klebsiella & 59 & $22.52 \%$ \\
\hline Staph epidermidis & 35 & $13.35 \%$ \\
\hline Staph aureus & 32 & $12.12 \%$ \\
\hline Proteus spp & 12 & $4.58 \%$ \\
\hline Pseudomonas & 8 & $3.05 \%$ \\
\hline Entrococci & 6 & $2.29 \%$ \\
\hline Corynebacteria & 4 & $1.53 \%$ \\
\hline Total & 262 & $100 \%$ \\
\hline
\end{tabular}

UTI and asymptomatic bacteriuria are common in the elderly, most often due to Escherichia coli (E. coli) colonization. (Figure 1) for example, in one study the prevalence of bacteriuria in an elderly ambulatory population was $18 \%$ in women and $6 \%$ in men. The prevalence increases in women, with age and institutionalization Boscia JA et al. ${ }^{11}$ The study was done on 10 groups of antibiotics with different mode of actions. Escherichia coli is the commonest cause of urinary tract infections especially in children, it was isolated from 106 $(40.46 \%)$ cases with clinically suspected UTI. The pattern of antibiotic sensitivity for that isolated Escherichia coli strains from urine samples was as follows: Ampicillin 9.4\%, Amoxycillin 70.8\%, Nalidixic acid $67.9 \%$, ceftriaxon $73.6 \%$, sulpha methoxazol + trimethoprim $25.5 \%$, cephlothin $21.7 \%$, ciprofloxacillin $85.5 \%$, Nitrofuranatian $71.7 \%$, chloramphenicol $48.1 \%$, Doxacycline $21.7 \%$ (Table 3 ).

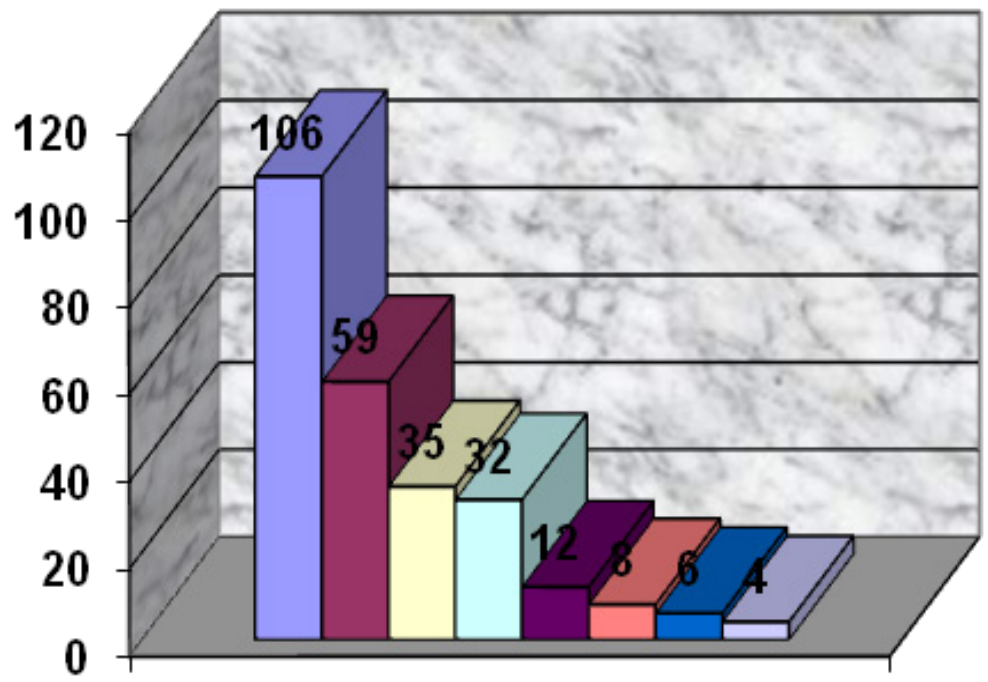

No. of positive culture cases

Figure I No of positive culture cases. 
Table 3 Shows antibiotics sensitivity/resistance pattern for isolated Escherichia coli strains from urine samples

\begin{tabular}{|l|l|l|l|l|l|}
\hline & Antibiotic & Frequency & Resistance & Sensitive & Resistance \\
\cline { 3 - 6 } & Sensitive & Resistin & $9.4 \%$ & $90.6 \%$ \\
\hline 2 & Ampicillin (AMP) & 10 & 96 & $70.8 \%$ & $29.2 \%$ \\
\hline 3 & Nalidixic acid (NA) & 75 & 31 & $67.9 \%$ & $32.1 \%$ \\
\hline 4 & Ceftriaxone (CRO) & 72 & 34 & $73.6 \%$ & $26.4 \%$ \\
\hline 5 & $\begin{array}{l}\text { Sulphamethoxazol + } \\
\text { Trimethoprim(SXT) }\end{array}$ & 78 & 28 & $25.5 \%$ & $74.5 \%$ \\
\hline 6 & Cephalothin (KF) & 27 & 79 & $21.7 \%$ & $78.3 \%$ \\
\hline 7 & Ciprofloxacin (CIP) & 23 & 83 & $85.8 \%$ & $14.2 \%$ \\
\hline 8 & Nitrofurantion (F) & 91 & 15 & $71.7 \%$ & $28.3 \%$ \\
\hline 9 & Chloramphenicol (C) & 76 & 30 & $48.1 \%$ & $51.9 \%$ \\
\hline 10 & Doxacycline "DO" & 51 & 55 & $21.7 \%$ & $78.3 \%$ \\
\hline & Total & 23 & 83 & & \\
\hline
\end{tabular}

Klebsiella organisms are Gram negative, non motile, capsulated bacilli represented $59(22.52 \%)$ from all isolated cases. Its pattern of antibiotic sensitivity was as follows: Ampicillin 0\%, Amoxycillin $71.2 \%$, Nalidixic acid 59.3\%, Ceftriaxon $67.8 \%$, Sulphamethoxazol+Trimethoprim 20.3\%, Cephlothin 20.3\%, Ciprofloxacillin $71.2 \%$, Nitrofuranation $52.5 \%$, Chloramphenicol $57.6 \%$, Doxacycline $25.4 \%$ (Table 4). Klebsiella organisms are Gram negative, non motile, capsulated bacilli represented $59(22.52 \%)$ from all isolated cases. Its pattern of antibiotic sensitivity was as follows: Ampicillin 0\%, Amoxycillin 71.2\%, Nalidixic acid 59.3\%, Ceftriaxon $67.8 \%$,
Sulphamethox-azol+Trimethoprim 20.3\%， Cephlothin 20.3\%, Ciprofloxacillin $71.2 \%$, Nitrofuranation $52.5 \%$, Chloramphenicol $57.6 \%$, Doxacycline $25.4 \%$ (Table 5). Staphylococcus epidermidis are Gram positive cocci arranged in clusters, Novobiocin sensitive and coagulase negative were isolated from 35 (13.35\%) cases with UTIs. Its pattern of antibiotic sensitivity was as follows Ampicillin $8.5 \%$, Amoxycillin $82.8 \%$, Nalidixic acid $40.0 \%$, Ceftriaxon $42.8 \%$, Sulphamethoxazol + Trimethoprim 27\%, Cephlothin 83.8\%, Ciproflxacillin $\mathrm{Hcl} 81.1 \%$, Nitrofuranatian $78.4 \%$, Chloramphenicol $20 \%$, Doxacycline $31.4 \%$ (Table 6).

Table 4 Shows antibiotic sensitivity/resistance pattern for isolated Klebsiella from urine samples

\begin{tabular}{|l|l|l|l|l|l|}
\hline & \multirow{2}{*}{ Antibiotic } & Frequency & $\%$ & Resistance \\
\cline { 3 - 6 } & Sensitive & Resistance & Sensitive & $100.0 \%$ \\
\hline 1 & Ampicillin (AMP) & 0 & 59 & $0.00 \%$ & $28.8 \%$ \\
\hline 2 & Amoxycillin (AMC) & 42 & 17 & $71.2 \%$ & $40.7 \%$ \\
\hline 3 & Nalidixic acid (NA) & 35 & 24 & $59.3 \%$ & $32.2 \%$ \\
\hline 4 & Ceftriaxone (CRO) & 40 & 19 & $67.8 \%$ & $79.7 \%$ \\
\hline 5 & Sulphamethoxazol + & 12 & 47 & $20.3 \%$ & $79.7 \%$ \\
\hline 6 & Trimethoprim(SXT) & 12 & 47 & $20.3 \%$ & $28.8 \%$ \\
\hline 7 & Cephalothin (KF) & 12 & 17 & $71.2 \%$ & $47.5 \%$ \\
\hline 8 & Ciprofloxacin (CIP) & 42 & 28 & $52.5 \%$ & $42.4 \%$ \\
\hline 9 & Chloramphenicol (C) & 31 & 25 & $57.6 \%$ & $74.6 \%$ \\
\hline 10 & Doxacycline "DO" & 34 & 44 & $25.4 \%$ & \\
\hline & Total & 15 & & & \\
\hline
\end{tabular}

Table 5 Shows antibiotic sensitivity/resistance pattern for isolated Staph aureus from urine samples

\begin{tabular}{|c|c|c|c|c|c|}
\hline & \multirow{2}{*}{ Antibiotic } & \multicolumn{2}{|c|}{ Frequency } & \multicolumn{2}{|l|}{$\%$} \\
\hline & & Sensitive & Resistance & Sensitive & Resistance \\
\hline I & Ampicillin (AMP) & 3 & 29 & $9.4 \%$ & $90.6 \%$ \\
\hline 2 & Amoxycillin (AMC) & 23 & 9 & $71.9 \%$ & $28.1 \%$ \\
\hline 3 & Nalidixic acid (NA) & 24 & 8 & $75.0 \%$ & $25.0 \%$ \\
\hline 4 & Ceftriaxone (CRO) & 13 & 19 & $40.6 \%$ & $59.4 \%$ \\
\hline 5 & $\begin{array}{l}\text { Sulphamethoxazol + } \\
\text { Trimethoprim(SXT) }\end{array}$ & 5 & 27 & $15.6 \%$ & $84.4 \%$ \\
\hline 6 & Cephalothin (KF) & 17 & 15 & $53.1 \%$ & $46.9 \%$ \\
\hline 7 & Ciprofloxacin (CIP) & 24 & 8 & $75.0 \%$ & $25.0 \%$ \\
\hline 8 & Nitrofurantion $(\mathrm{F})$ & 20 & 12 & $62.5 \%$ & $37.5 \%$ \\
\hline 9 & Chloramphenicol (C) & 15 & 17 & $46.9 \%$ & $53.1 \%$ \\
\hline \multirow[t]{2}{*}{10} & Doxacycline "DO" & 12 & 20 & $37.5 \%$ & $62.5 \%$ \\
\hline & Total & 32 & & & \\
\hline
\end{tabular}


Table 6 Shows antibiotic sensitivity/resistance pattern for isolated Staph Epidermidis from urine samples

\begin{tabular}{|c|c|c|c|c|c|}
\hline & \multirow{2}{*}{ Antibiotic } & \multicolumn{2}{|c|}{ Frequency } & \multicolumn{2}{|l|}{$\%$} \\
\hline & & Sensitive & Resistance & Sensitive & Resistance \\
\hline 1 & Ampicillin (AMP) & 3 & 32 & $8.5 \%$ & $91.5 \%$ \\
\hline 3 & Nalidixic acid (NA) & 14 & 21 & $40.0 \%$ & $60.0 \%$ \\
\hline 4 & Ceftriaxone (CRO) & 15 & 20 & $42.8 \%$ & $57.2 \%$ \\
\hline 6 & Cephalothin (KF) & 29 & 6 & $83.8 \%$ & $16.2 \%$ \\
\hline 7 & Ciprofloxacin (CIP) & 28 & 7 & $81.1 \%$ & $18.9 \%$ \\
\hline 8 & Nitrofurantion (F) & 27 & 8 & $78.4 \%$ & $21.6 \%$ \\
\hline 9 & Chloramphenicol (C) & 7 & 28 & $20.0 \%$ & $80.0 \%$ \\
\hline
\end{tabular}

Enterococci are Gram positive cocci arranged in short chains or mostly in pairs, and catalase negative were isolated from $6(2.29 \%)$ cases with UTIs. Its pattern of antibiotic sensitivity was as follows Ampicillin 83.3\%, Amoxycillin 100\%, Nalidixic acid 66.7\%, Ceftriaxon 100\%, Sulpha methoxazol + Trimethoprim 0\%, Cephlothin 83.3\%, Ciprofloxacillin 50\%, Nitrofuration 33.3\%, Chlooramphenicol 50\%, Doxacycline 0\% (Table 7). Pseudomonas spp are motile Gram negative bacilli, strictly aerobic and non-spore forming bacteria. The only species pathogenic for human, pyocyanin, which give the colour to "blue pus", were isolated from $8(3.05 \%)$ cases with UTIs. Its pattern of antibiotic sensitivity/resistance as follows: Ampicillin 0\%, Amoxycillin 0\%, Nalidixic acid 12.5\%, Ceftriaxon 25\%, Sulpha methoxazol + Trimethoprim 100\%, Cephlothin 0\%, Ciprofloxacillin 87.5\%, Nitrofuration 0\%, Chlooramphenicol 0\%, Doxacycline 12.5\% (Table 8). Proteus spp. are enterobacteriaceae characterized by swarming motility and urease production, represent $12(4.58 \%)$ from all isolated cases. Its pattern of antibiotic sensitivity was as follows: Ampicillin 0\%, Amoxycillin 41.6\%, Nalidixic asid 50\%, Ceftriaxon 66.6\%, Sulphamethoxazol + Trimethoprim 50\%, Cephlothin 16.6\%, Ciprofloxacillin 75\%, Nitrofuranatian 66.6\%, Chloramphenicol 41.6\%, Doxacycline 25\% (Table 9).

Table 7 Shows antibiotic sensitivity/resistance pattern for isolated Enterococci from urine samples

\begin{tabular}{|c|c|c|c|c|c|}
\hline & \multirow{2}{*}{ Antibiotic } & \multicolumn{2}{|c|}{ Frequency } & \multicolumn{2}{|l|}{$\%$} \\
\hline & & Sensitive & Resistance & Sensitive & Resistance \\
\hline I & Ampicillin (AMP) & 5 & 1 & $83.3 \%$ & $16.7 \%$ \\
\hline 2 & Amoxycillin (AMC) & 6 & 0 & $100.0 \%$ & $0.0 \%$ \\
\hline 3 & Nalidixic acid (NA) & 4 & 2 & $66.7 \%$ & $33.3 \%$ \\
\hline 4 & Ceftriaxone (CRO) & 6 & 0 & $100.0 \%$ & $0.0 \%$ \\
\hline 5 & $\begin{array}{l}\text { Sulphamethoxazol + } \\
\text { Trimethoprim(SXT) }\end{array}$ & 0 & 6 & $0.0 \%$ & $100.0 \%$ \\
\hline 6 & Cephalothin (KF) & 5 & 1 & $83.3 \%$ & $16.7 \%$ \\
\hline 7 & Ciprofloxacin (CIP) & 3 & 3 & $50.0 \%$ & $50.0 \%$ \\
\hline 8 & Nitrofurantion (F) & 2 & 4 & $33.3 \%$ & $66.7 \%$ \\
\hline 9 & Chloramphenicol (C) & 3 & 3 & $50.0 \%$ & $50.0 \%$ \\
\hline \multirow[t]{2}{*}{10} & Doxacycline "DO" & 0 & 6 & $0.0 \%$ & $100.0 \%$ \\
\hline & Total & 6 & & & \\
\hline
\end{tabular}

Table 8 Shows antibiotic sensitivity/resistance pattern for isolated Pseudomonas from urine samples

\begin{tabular}{|c|c|c|c|c|c|}
\hline & \multirow{2}{*}{ Antibiotic } & \multicolumn{2}{|c|}{ Frequency } & \multicolumn{2}{|l|}{$\%$} \\
\hline & & Sensitive & Resistance & Sensitive & Resistance \\
\hline 1 & Ampicillin (AMP) & 0 & 8 & $00.0 \%$ & $100.0 \%$ \\
\hline 3 & Nalidixic acid (NA) & I & 7 & $12.5 \%$ & $87.5 \%$ \\
\hline 4 & Ceftriaxone (CRO) & 2 & 6 & $25.0 \%$ & $75.0 \%$ \\
\hline 6 & Cephalothin (KF) & 0 & 8 & $0.0 \%$ & $100.0 \%$ \\
\hline 7 & Ciprofloxacin (CIP) & 7 & I & $87.5 \%$ & $12.5 \%$ \\
\hline 8 & Nitrofurantion (F) & 0 & 8 & $0.0 \%$ & $100.0 \%$ \\
\hline 9 & Chloramphenicol (C) & 0 & 8 & $0.0 \%$ & $100.0 \%$ \\
\hline
\end{tabular}


Table 9 Shows antibiotic sensitivity/resistance pattern for isolated Proteus spp from urine samples

\begin{tabular}{|l|l|l|l|l|l|}
\hline & \multirow{2}{*}{ Antibiotic } & Frequency & $\%$ & Resistance \\
\cline { 3 - 6 } & Sensitive & Resistance & Sensitive & $00.0 \%$ & $100.0 \%$ \\
\hline 1 & Ampicillin (AMP) & 0 & 12 & $41.6 \%$ & $58.4 \%$ \\
\hline 2 & Amoxycillin (AMC) & 5 & 7 & $50.0 \%$ & $50.0 \%$ \\
\hline 3 & Nalidixic acid (NA) & 6 & 6 & $66.6 \%$ & $33.3 \%$ \\
\hline 4 & Ceftriaxone (CRO) & 8 & 4 & $50.0 \%$ & $50.0 \%$ \\
\hline 5 & $\begin{array}{l}\text { Sulphamethoxazol + } \\
\text { Trimethoprim(SXT) }\end{array}$ & 6 & 6 & $16.6 \%$ & $83.4 \%$ \\
\hline 6 & Cephalothin (KF) & 2 & 10 & $75.0 \%$ & $25.0 \%$ \\
\hline 7 & Ciprofloxacin (CIP) & 9 & 3 & $66.6 \%$ & $33.4 \%$ \\
\hline 8 & Nitrofurantion (F) & 8 & 4 & $41.6 \%$ & $58.4 \%$ \\
\hline 9 & Chloramphenicol (C) & 5 & 7 & $25.0 \%$ & $75.0 \%$ \\
\hline 10 & Doxacycline "DO" & 3 & 9 & & \\
\hline & Total & 12 & & & \\
\hline
\end{tabular}

Corynebacteria are Gram positive aerobic, non motile, non spore forming bacilli represent the lowest isolated cases in the study 4 $(1.53 \%)$ from all isolated cases. Its pattern of antibiotic sensitivity was as follows: Ampicillin 75\%, Amoxycillin 75\%, Nalidixic acid $75 \%$, Ceftriaxon 25\%, Sulphamethoxazol + Trimethoprim 50\%, Cephlothin 100\%, Ciprofloxacillin 100\%, Nitrofuranatian 100\%, Chloramphenicol 0\%, Doxacycline 75\% (Table 10). After detection of antibiotic sensitivity/resistance patterns for every individual isolated pathogenic bacteria from urine samples (262). Table 11 is a crude table showing relationship between antibiotic sensitivity/resistance pattern for all isolated bacteria and number of cases. It shows the antibiotic sensitivity patterns for all isolated pathogenic bacteria as follows: Ampicillin 9.2\%, Amoxycillin 70.2\%, Nalidixic asid $60.3 \%$, Ceftriaxon $61.8 \%$, Sulphamethoxazol + Trimethoprim $26.3 \%$, Cephlothin 35.5\%, Ciprofloxacillin 80.2\%, Nitrofuranatian 64.5\%, Chloramphenicol $43.1 \%$, Doxacycline $26.3 \%$.

Table 10 Shows antibiotic sensitivity/resistance pattern for isolated Corynebacteria from urine samples

\begin{tabular}{|c|c|c|c|c|c|}
\hline & \multirow{2}{*}{ Antibiotic } & \multicolumn{2}{|c|}{ Frequency } & \multicolumn{2}{|l|}{$\%$} \\
\hline & & Sensitive & Resistance & Sensitive & Resistance \\
\hline I & Ampicillin (AMP) & 3 & 1 & $75.0 \%$ & $25.0 \%$ \\
\hline 2 & Amoxycillin (AMC) & 3 & 1 & $75.0 \%$ & $25.0 \%$ \\
\hline 3 & Nalidixic acid (NA) & 3 & 1 & $75.0 \%$ & $25.0 \%$ \\
\hline 4 & Ceftriaxone (CRO) & 1 & 3 & $25.0 \%$ & $75.0 \%$ \\
\hline 5 & $\begin{array}{l}\text { Sulphamethoxazol + } \\
\text { Trimethoprim(SXT) }\end{array}$ & 2 & 2 & $50.0 \%$ & $50.0 \%$ \\
\hline 6 & Cephalothin (KF) & 4 & 0 & $100.0 \%$ & $0.0 \%$ \\
\hline 7 & Ciprofloxacin (CIP) & 4 & 0 & $100.0 \%$ & $0.0 \%$ \\
\hline 8 & Nitrofurantion (F) & 4 & 0 & $100.0 \%$ & $0.0 \%$ \\
\hline 9 & Chloramphenicol (C) & 0 & 4 & $0.0 \%$ & $100.0 \%$ \\
\hline \multirow[t]{2}{*}{10} & Doxacycline "DO" & 3 & 1 & $75.0 \%$ & $25.0 \%$ \\
\hline & Total & 4 & & & \\
\hline
\end{tabular}

Table I I Shows antibiotic sensitivity/resistance patterns for all isolated pathogenic bacteria from urine samples

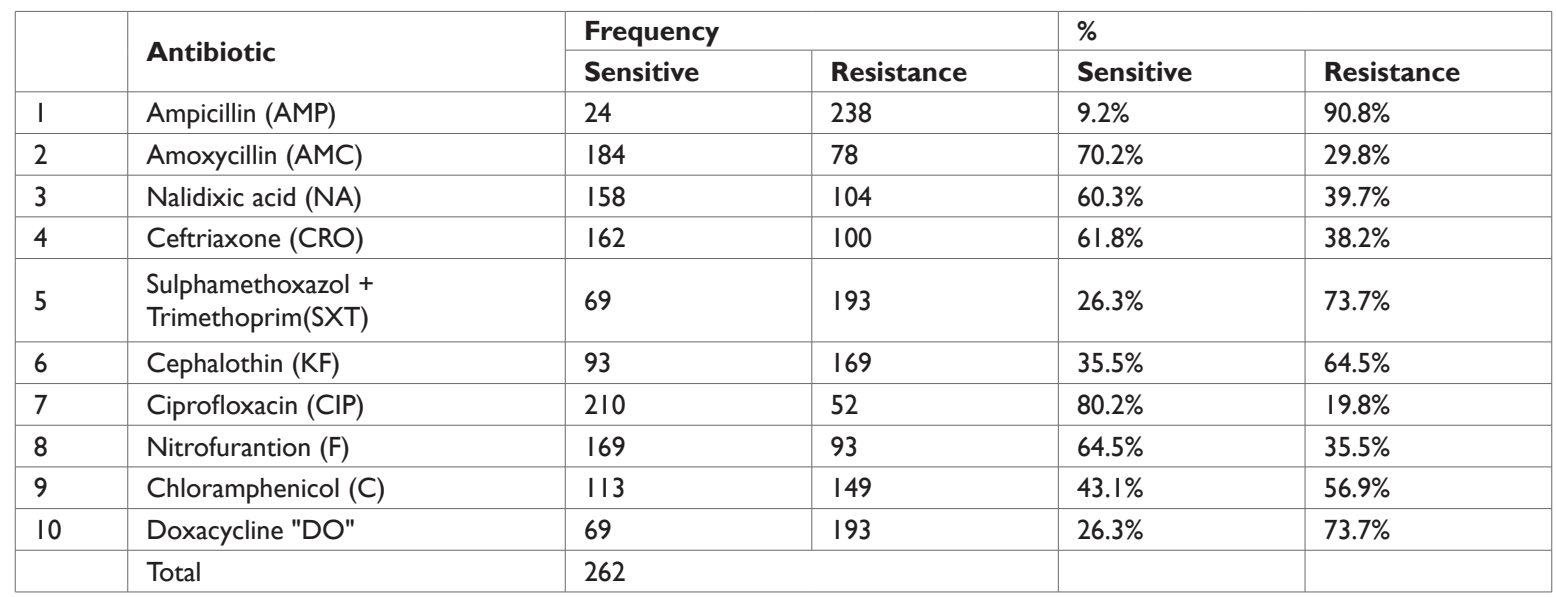




\section{Discussion}

This study indicates the following results:

I. Urinary tract infection is a major cause of morbidity especially in young children, $173(66 \%)$ out of 262 cases showed positive growth on bacterial cultures, received during the four months period of study.

II. Trends in choice of antibiotic treatment may change depending on locally determined resistances, common pathogens and cost issues.

In El Batnan Medical Center, during 2003 and 2004 in the UTI patients we found that $E$ Coli was the most common pathogen $40.46 \%$ (106/262), and was most sensitive to Ciprofloxacin (85.8\%) with noticeable resistance to Ampicillin (90.6\%resistance). Klebsiella was the second commonest pathogen 22.5\% (59/262) against which Ciprofloxacin and Amoxicillin were most sensitive (71.2\%) for both, with clear resistance to Ampicillin (100\%) and Cephalothin (79.7\%). Staph epidermidis was the third revealed pathogen $13.35 \%(35 / 262)$ against which Amoxycillin and Cephalothin (82.8\%), (83.8\%) respectively were most sensitive.

Staph aureus represented $12.21 \%$ (32/262) of cases, Nalidixic acid and, Ciprofloxacin were most sensitive $75 \%$ with clear resistance to Ampicillin (90.6\%). Enterococcus 2.29\% (6/262) of cases, Amoxycillin and Ceftriaxon were the most sensitive 100\% while Doxacycline was resistance in all cases $100 \%$. Pseudomonas $3.05 \%(8 / 262)$ of cases. Sulphamethoxazol + trimethoprim (CoTrimoxazole) were most sensitive in all cases $100 \%$, while Ampicillin, Amoxycillin, Cephalothin, Nitrofurantoin and Chloramphenicl were most resistance in all cases $100 \%$. Proteus spp. represented $4.58 \%$ (12/262) of cases. Ciprofloxacin was sensitive in $75 \%$ of cases, while Ampicillin was resistant in all cases $100 \%$. Corynebacteria was the least common organism seen in UTIs $1.6 \%$ of cases $(4 / 262)$. Cephalothin, Ciprofloxacin and Nitrofurantian sensitivity were $100 \%$ in all cases, while Chloramphenicol was resistant in all cases $100 \%$, Ceftriaxon was only sensitive in $75 \%$ of cases.

III. The most sensitive antibiotics to all isolated bacteria were Ciprofloxacin $80.2 \%$ and Amoxycillin (70.2\%), while Ampicillin was the least sensitive only in $9.2 \%$, among all UTIs cases. Although many clinicians favour the use of Ampicillin or Co-amoxiclav believing it will have a higher 'hit rate', we concluded that we should be using less Ampicillin and Coamoxiclav for empirical treatment. We recommended the use of more Ciprofloxacin for empirical treatment, with three day course or Ceftriaxone (in case of the parenteral route) ${ }^{12,13}$. Certain differences may change our treatment policies, such as effect of antibiotic side effects, particularly relating to renal function, altered antibiotic resistances compared to younger populations. Again, few studies addressing these issues exist, and decisions are made anecdotally.

IV. The following antibiotics are the most sensitive antibiotics in all UTIs cases, firstly, Ciprofloxacin $80.2 \%$, Amoxicillin $70.2 \%$, Nitrofurantion $64.5 \%$, and Ceftriaxon $61 \%$.

\section{Conclusion}

In the notable absence of studies in the treatment of uncomplicated UTI we recommend empirical antimicrobial treatment based on local sensitivities which should be changed once the local pattern of sensitivities is known.

\section{Recommendations}

Antibiotic therapy certain rules have to be applied:

i. Do not give antibiotic without good evidence of an infection treatable with the antibiotic.

ii. Stop the antibiotic after 5 days if the patient is better.

iii. Do not give large doses of powerful antibiotic or combination of antibiotic unless the patient is very sick, as it eliminates the normal microbial flora and lead to further infection.

\section{References}

1. Royal College of General Practitioners Birmingham Research Unit. Guidelines for the management of acute urinary tract infection in childhood. J R Coll Physicians Lond. 2004;25(1):36-42.

2. Nordenstam GR, Brandeberg CA, Oden AS, et al. Bacteriuria and mortality in an elderly population. NEJM. 1986;314(18):1152-1156.

3. Coulthard MG, Lambert HJ, Keir MJ. Occurrence of renal scars in children after their first referral for urinary tract infection. $B M J$. 1997;315(7113):918-919.

4. Jadresic L, Cartwright $\mathrm{K}$, Cowie N. Investigation of urinary tract infection in childhood. British Medical Journal. 1993;307:761-764.

5. Coulthard MG, Vernon SJ, Lambert HJ, et al. A nurse led education and direct access service for the management of urinary tract infections in children: prospective controlled trial. BMJ. 2003;327(7416):656.

6. Klein RS. Criteria for the diagnosis of urinary tract infection. Curr Opin Nephrol and Hypertension. 1994;3:652-655.

7. Liaw LCT, Nayar DN, Pedler SJ, et al. Home collection of urine for culture from infants by three methods: survey of parents' preferences and bacterial contamination rates. BMJ. 2000;320(7245):1312-1313.

8. Abla M El-Mishad nd Adelberg's, Professor of medical microbiology and immunology, Faculty of Medicine, Cairo University Manual of Practical Microbiology, pp. 13, 94, 104, 106, 78, 81, 86,113-112.

9. Munima Shaikh, Baqi S Naqvi, Zafar A Mahmood, et al. Medical Journal of Islamic World Academy of sciences. 2005;15: 129-133.

10. Jawetz, Melnick, Adelgerg's. Review of Medical Microbiology. (23 $3^{\text {rd }}$ edn), 2003;pp.433-440.

11. Boscia JA, Kobasa WD, Knight RA, et al. Epidemiology of bacteriuria in an elderly ambulatory population. Am J Med. 1986;80(2):208-214.

12. Van der Voort J, Edwards A, Roberts R et al. The struggle to diagnose UTI in children under two in primary care. Family Practice. 1997;14:44-48.

13. Iravani $\mathrm{A}$, Tice $\mathrm{AD}, \mathrm{McCarthy} \mathrm{J}$, et al. Short-course ciprofloxacin treatment of acute uncomplicated urinary tract infection in women. The minimum effective dose. The Urinary Tract Infection Study Group. Arch Int Med. 1995;155(5):485-494. 